\title{
31 Polypenmanagement
}

Andreas Probst, Helmut Messmann

\subsection{Definition}

Ursprünglich leitet sich der Begriff „Polyp“vom griechischen Wort polýpous (vielfüßig) ab und impliziert eine gestielte Läsion. Im weiteren Sinne definiert sich ein Kolonpolyp als Gewebevorwölbung, die aus der Kolonschleimhaut heraus in das Darmlumen ragt. Die Bezeichnung Polyp beinhaltet keine weitere Festlegung zu Form (gestielt, breitbasig), Morphologie (zottig, glatt), Gewebeart (neoplastisch, nicht-neoplastisch) und Dignität (benigne, maligne). Histopathologisch wird zwischen epithelialen und nicht-epithelialen sowie zwischen neoplastischen und nicht-neoplastischen Polypen unterschieden. Das folgende Kapitel beschränkt sich auf die Diagnostik und Therapie epithelialer Polypen (klassische Adenome, serratierte Läsionen, hyperplastische Polypen, Frühkarzinome).

\subsubsection{Adenome}

Der häufigste Kolonpolyp ist das benigne Adenom. In einer großen Studie zur Vorsorgekoloskopie wurden bei 32,9\% aller Koloskopien Polypen gefunden; bei 20,7\% handelte es sich um Adenome, bei 0,9\% um ein Karzinom. 56,8\% der Patienten mit Polypen hatten einen Polypen, 37,3\% hatten 2-4 Polypen und 5,9\% hatten mehr als 4 Polypen. Histologisch werden tubuläre, tubulovillöse und villöse Adenome unterschieden. Zusätzlich wird eine histologische Graduierung der Dysplasie innerhalb eines Adenoms vorgenommen (LGIEN versus HGIEN). Als fortgeschrittenes Adenom (advanced adenoma) bzw. high-risk-Adenom gilt ein Adenom bei einer Größe >10 mm, bei Vorliegen einer tubulovillösen oder villösen Histologie sowie bei Nachweis einer hochgradigen intraepithelialen Neoplasie (HGIEN). Die potentielle maligne Entartung von Kolonadenomen zum kolorektalen Karzinom (,adenoma-adenocarcinomasequence“) ist seit den 1950er Jahren bekannt.

\subsubsection{Serratierte Adenome}

Neben der klassischen Adenom-Karzinom-Sequenz stellt der sogenannte „,serratierte Karzinogeneseweg“ einen weiteren Mechanismus der kolorektalen Krebsentstehung dar. Hierbei entwickeln sich kolorektale Karzinome aus primär benignen ,serratierten Adenomen“. Unterschieden werden sessile serratierte Adenome (SSA), traditionelle serratierte Adenome (TSA) und gemischte Polypen (mixed polyps). Gemischte Polypen enthalten sowohl hyperplastische/serratierte als auch klassische Adenomanteile 
(tubulär, tubulovillös, villös). Serratierte Läsionen sind gegenüber klassischen Adenomen seltener.

Die aktuelle WHO-Klassifikation unterteilt „serrated lesions“ in 3 Gruppen: SSA, TSA und hyperplastische Polypen (HP), die histologisch ebenfalls „serratiert“ erscheinen. Hinsichtlich Morphologie, Lokalisation und Entartungspotential unterscheiden sich die drei Gruppen (Tab. 31.1).

Tab. 31.1: Einteilung serratierter Kolonpolypen.

\begin{tabular}{llllll}
\hline & Form & Größe & Häufigkeit & $\begin{array}{l}\text { Lokalisation im } \\
\text { Kolon }\end{array}$ & $\begin{array}{l}\text { Malignes } \\
\text { Potenzial }\end{array}$ \\
\hline HP & flach, sessil & klein (oft $<5 \mathrm{~mm})$ & sehr häufig & links & nein \\
\hline SSA & flach, sessil & größer als HP & häufig & rechts & ja \\
\hline TSA & flach, gestielt & größer als HP & selten & links & ja \\
\hline
\end{tabular}

\subsection{Diagnostik/Klassifikation kolorektaler Polypen}

\subsubsection{Wachstumsform}

Als grobe Einteilung anhand der makroskopisch klassifizierbaren Wachstumsform erfolgte lange Zeit eine einfache Unterscheidung in gestielte, taillierte und breitbasig wachsende (sessile) Polypen. Mit zunehmender Erkennung flacher Läsionen erfolgte eine Differenzierung in polypoide Läsionen, flache Läsionen und unter das Schleimhautniveau eingesenkte Läsionen, was 2005 als Paris-Klassifikation zusammengefasst wurde. Die Paris-Klassifikation gilt prinzipiell im gesamten Gastrointestinaltrakt und unterteilt polypoide Läsionen in zwei Subgruppen (gestielt versus sessil) sowie flache Läsionen in drei Subgruppen (flach-erhaben versus ausschließlich flach versus flach-eingesenkt) (Abb. 31.1).

Die Paris-Klassifikation erlaubt die Beschreibung einfacher, aber auch komplexer Läsionen (z. B. Paris-Typ IIa + IIc bei Läsionen mit flach erhabenen und eingesenkten Anteilen) (Abb. 31.2).

Für flache Läsionen (Paris-Typ-II-Läsionen) mit einem Durchmesser von mehr als $10 \mathrm{~mm}$ wurde zusätzlich die Klassifikation der „laterally spreading tumors“ (LST) eingeführt. Anhand der Polypenmorphologie wird zwischen „granular type“ (LST-G) und „non-granular type“ (LST-NG) unterschieden. Beim LST-G zeigt sich ein Aggregat nebeneinanderliegender Knötchen, während die LST-NG eine weniger strukturierte Oberfläche aufweisen. LST-G werden weiter unterteilt in homogene flache Läsionen (,homogenous type“) sowie Läsionen mit zusätzlichen Knoten („nodular mixed type“). Beim LST-NG werden flach erhabene (,flat elevated“) von eingesenkten Läsionen („pseudo depressed“) unterschieden. Die klinische Relevanz der LST-Klassifikation 
liegt in der unterschiedlichen Wahrscheinlichkeit einer malignen Entartung. In einer Publikation von Uraoka et al. wiesen 7\% der LST-G gegenüber $14 \%$ der LST-NG ein submukosainvasives Karzinom auf. In der LST-G-Gruppe war ein Malignitätsrisiko vor allem bei Nachweis polypoider Anteile (,nodular mixed type“) zu erkennen, bei LSTNG vor allem bei eingesenkter Morphologie („pseudo depressed“) (Abb. 31.2).
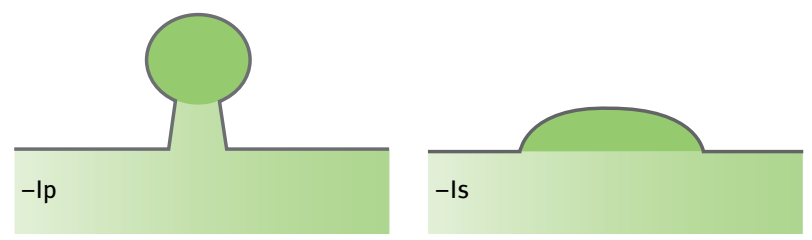

(a)
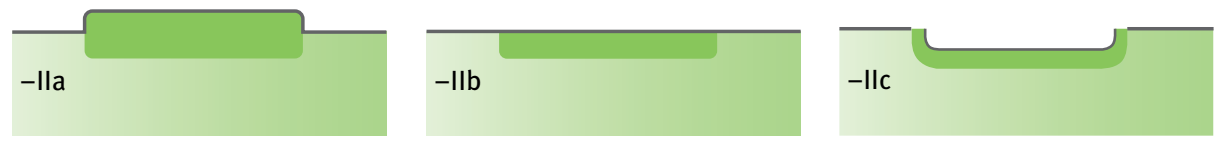

(b)

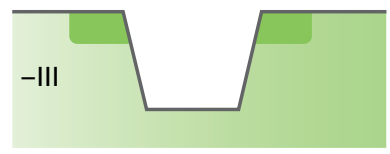

(c)

Abb. 31.1: Paris-Klassifikation oberflächlicher gastrointestinaler Neoplasien: Typ I= protruding, $\mathrm{I} \mathrm{p}=$ pedunculated, Is = sessile; Typ II = non protruding non excavated; Ila = slightly elevated, $\mathrm{Ilb}=$ completely flat, $\mathrm{Ilc}=$ slightly depressed; Typ III = excavated (ulzeriert).
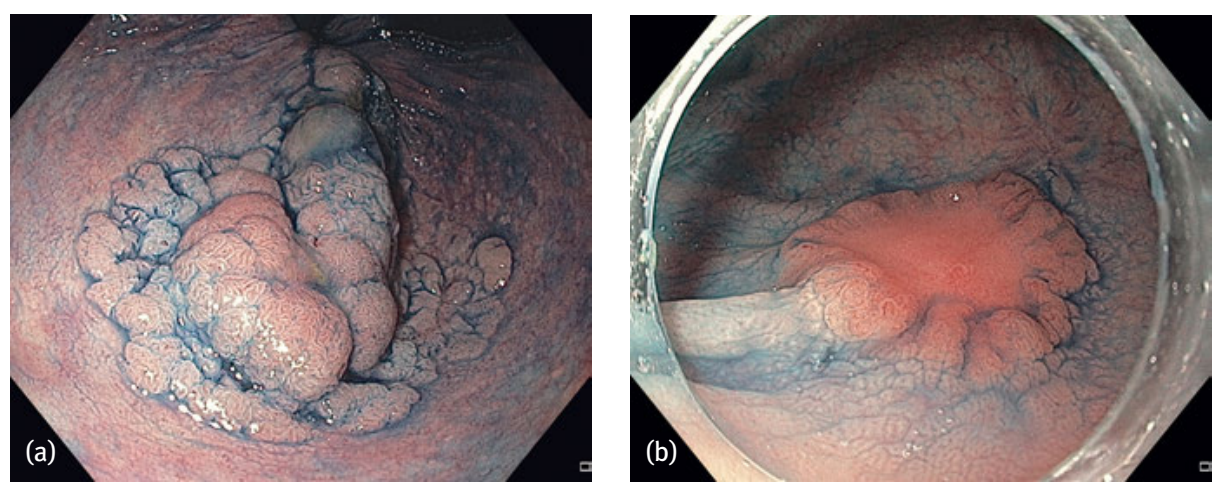

Abb. 31.2: Laterally spreading tumors (LST); Chromoendoskopie mit Indigokarmin (Histologie jeweils Adenom mit HGIEN). (a) LST granular type mit Knoten (LST-G „nodular mixed type“); (Paris 0-Ila + Is); (b) LST non granular type mit zentraler Einsenkung (LST-NG „pseudo depressed”) (Paris $0-I I a+I I c)$. 


\subsubsection{Feinbeurteilung der Oberflächen- und Gefäßarchitektur}

Nach Einteilung eines Polypen anhand der Paris- bzw. der LST-Klassifikation sollte eine Feinbeurteilung des Oberflächenreliefs und der Gefäßarchitektur erfolgen. Für die Beschreibung des Oberflächenreliefs führten Kudo et al. 1996 durch Kombination von Vergrößerungs- und Chromoendoskopie die „pit-pattern-Klassifikation“ ein, die 5 verschiedene Typen des Oberflächenreliefs unterscheidet. Auch hiermit kann eine morphologische Dignitätseinschätzung eines Polypen erfolgen. Neben der klassischen Chromoendoskopie wurden neue Technologien zur digitalen Strukturverstärkung entwickelt (NBI, BLI, FICE, i-scan), die neben der Feinbeurteilung des Oberflächenreliefs (surface pattern) eine zusätzliche Beurteilung der Gefäßarchitektur (vascular pattern) erlauben. Vor allem für NBI (narrow band imaging) wurden hierzu diverse Klassifikationen entwickelt; aktuell wird die japanische JNET-Klassifikation favorisiert (Tab. 31.2) (Abb. 31.3, 31.5, 31.6).

Tab.31.2: JNET-Klassifikation zur Dignitätsbeurteilung kolorektaler Neoplasien.

\begin{tabular}{|c|c|c|c|c|}
\hline JNET-Klassifikation & Typ 1 & Typ 2 A & Typ 2B & Typ 3 \\
\hline $\begin{array}{l}\text { Gefäßmuster } \\
\text { (NBI-Modus) }\end{array}$ & kein Gefäßnetz & regulär & irregulär & avaskulär \\
\hline $\begin{array}{l}\text { Oberflächenrelief } \\
\text { (NBI-Modus) }\end{array}$ & $\begin{array}{l}\text { regulär (punkt- } \\
\text { förmig, ähnlich der } \\
\text { normalen Mukosa) }\end{array}$ & $\begin{array}{l}\text { regulär (ver- } \\
\text { zweigt, tubu- } \\
\text { lär, netzartig) }\end{array}$ & irregulär & amorph \\
\hline $\begin{array}{l}\text { wahrscheinliche } \\
\text { Histologie }\end{array}$ & $\begin{array}{l}\text { hyperplastischer } \\
\text { Polyp oder serra- } \\
\text { tierte Läsion }\end{array}$ & $\begin{array}{l}\text { Adenom mit } \\
\text { leichter Dys- } \\
\text { plasie }\end{array}$ & $\begin{array}{l}\text { Adenom mit schwerer } \\
\text { Dysplasie oder Karzi- } \\
\text { nom mit oberflächlicher } \\
\text { Submukosainvasion }\end{array}$ & $\begin{array}{l}\text { Karzinom mit } \\
\text { mindestens } \\
\text { tiefer Sub- } \\
\text { mukosainva- } \\
\text { sion }\end{array}$ \\
\hline
\end{tabular}



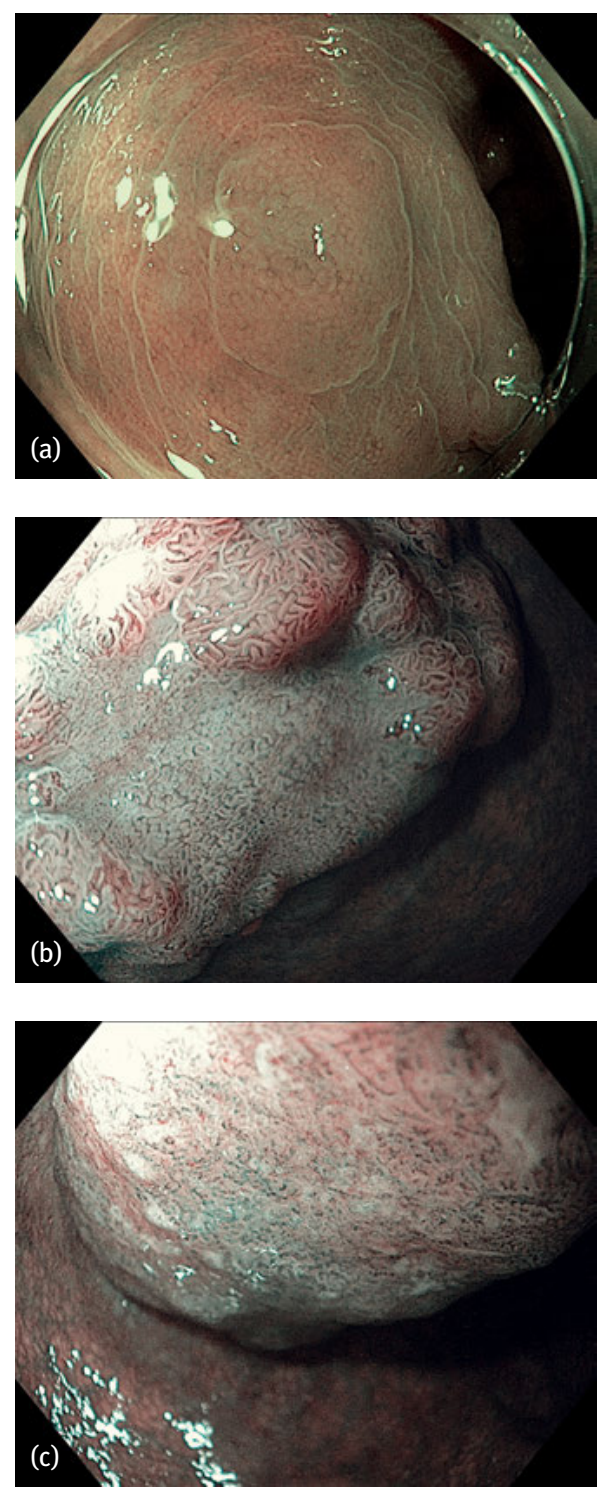

Abb. 31.3: Beurteilung von Kolonpolypen anhand der JNET-Klassifikation. (a) JNET 1, Histologie (Biopsie): hyperplastischer Polyp; (b) JNET 2 (am Rand JNET $2 \mathrm{~A}$, zentral im eingesenkten Areal JNET 2B), Histologie (ESD en bloc): Adenom mit schwerer Dysplasie; (c) JNET 3, Histologie (onkologische chirurgische Resektion mit Lymphknotendissektion): pT2 pN0.

\subsection{Endoskopische Resektionsverfahren kolorektaler Polypen}

Aufgrund ihres malignen Potenzials müssen alle konventionellen Adenome, alle SSA und alle TSA komplett entfernt werden; in aller Regel ist dies mittels ER möglich. Mittlerweile ist belegt, dass durch eine konsequente Entfernung von Adenomen die Inzidenz des kolorektalen Karzinoms längerfristig reduziert werden kann. Alleinig typische hyperplastische Polypen mit einem Durchmesser $<5 \mathrm{~mm}$ im Rektosigmoid dürfen belassen werden. 
Bei der endoskopischen Entfernung kolorektaler Polypen sollte immer eine komplette Entfernung der Läsion angestrebt werden. Für kleine Polypen $(\leq 5 \mathrm{~mm})$ ist eine Entfernung mittels Biopsiezange möglich, während bei einer Polypengröße von 6-9 mm eine Schlingenresektion ohne Hochfrequenzstrom empfohlen wird (cold snare). Für die Entfernung von Polypen $\geq 10 \mathrm{~mm}$ sind Resektionsverfahren unter Verwendung von Hochfrequenzstrom erforderlich.

\subsubsection{Endoskopische Polypektomie}

Bei der klassischen Polypektomie wird der Kolonpolyp mit einer Metallschlinge gefasst und durch Applikation von Hochfrequenzstrom reseziert. Die Polypektomie kann als Verfahren der Wahl bei gestielten Polypen betrachtet werden (Abb. 31.4). Für große Polypen (Stieldurchmesser $\geq 10 \mathrm{~mm}$ oder Polypenkopf $\geq 20 \mathrm{~mm}$ ) wird eine additive Blutungsprophylaxe vor der Resektion empfohlen (z. B. Injektion von Suprarenin 1:100.000 in den Stiel).
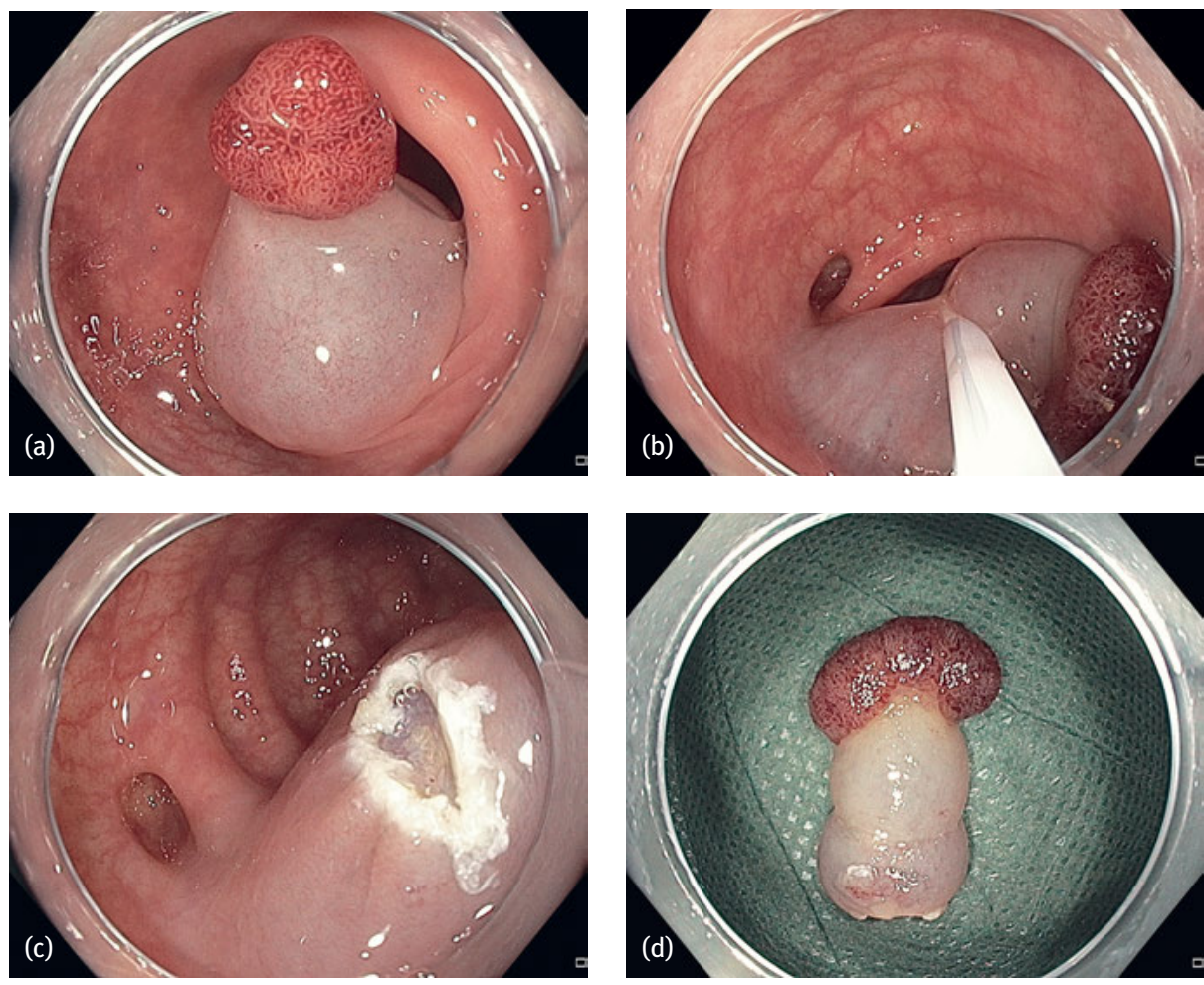

Abb. 31.4: Endoskopische Polypektomie eines gestielten Kolonpolypen. (a) Infiltration des Polypenstiels mit Suprarenin 1:100.000; (b) Um den Stiel platzierte Diathermieschlinge; (c) Resektionsulkus; (d) Resektat (Histologie: Adenom mit LGIEN). 


\subsubsection{Endoskopische Mukosaresektion (EMR)}

Die EMR kommt bei flächig wachsenden Läsionen zum Einsatz. Nach Injektion eines Flüssigkeitsdepots in die Submukosa wird die abzutragende Läsion angehoben (,lifting“), der Abstand zwischen Resektionslinie und Muskulatur vergrößert und damit die Perforationsgefahr im Rahmen der Resektion reduziert. Die Resektion erfolgt dann analog zur Polypektomie mittels Metallschlinge und Hochfrequenzstrom. Die Methode stellt das Standardresektionsverfahren für flache, mutmaßlich benigne Polypen mit einem Durchmesser $\geq 10 \mathrm{~mm}$ dar. Als Nachteil der EMR gilt die Limitation des Resektionsausmaßes durch die Größe der verwendeten Schlingen. Bei größeren Läsionen bzw. regelhaft bei Läsionen mit einem Durchmesser von $>20 \mathrm{~mm}$ muss die EMR als Resektion in mehreren Stücken erfolgen (piecemeal-Resektion pEMR). Bei einer pEMR erhöht sich das Risiko der inkompletten Resektion und das Rezidivrisiko (Abb. 31.5).
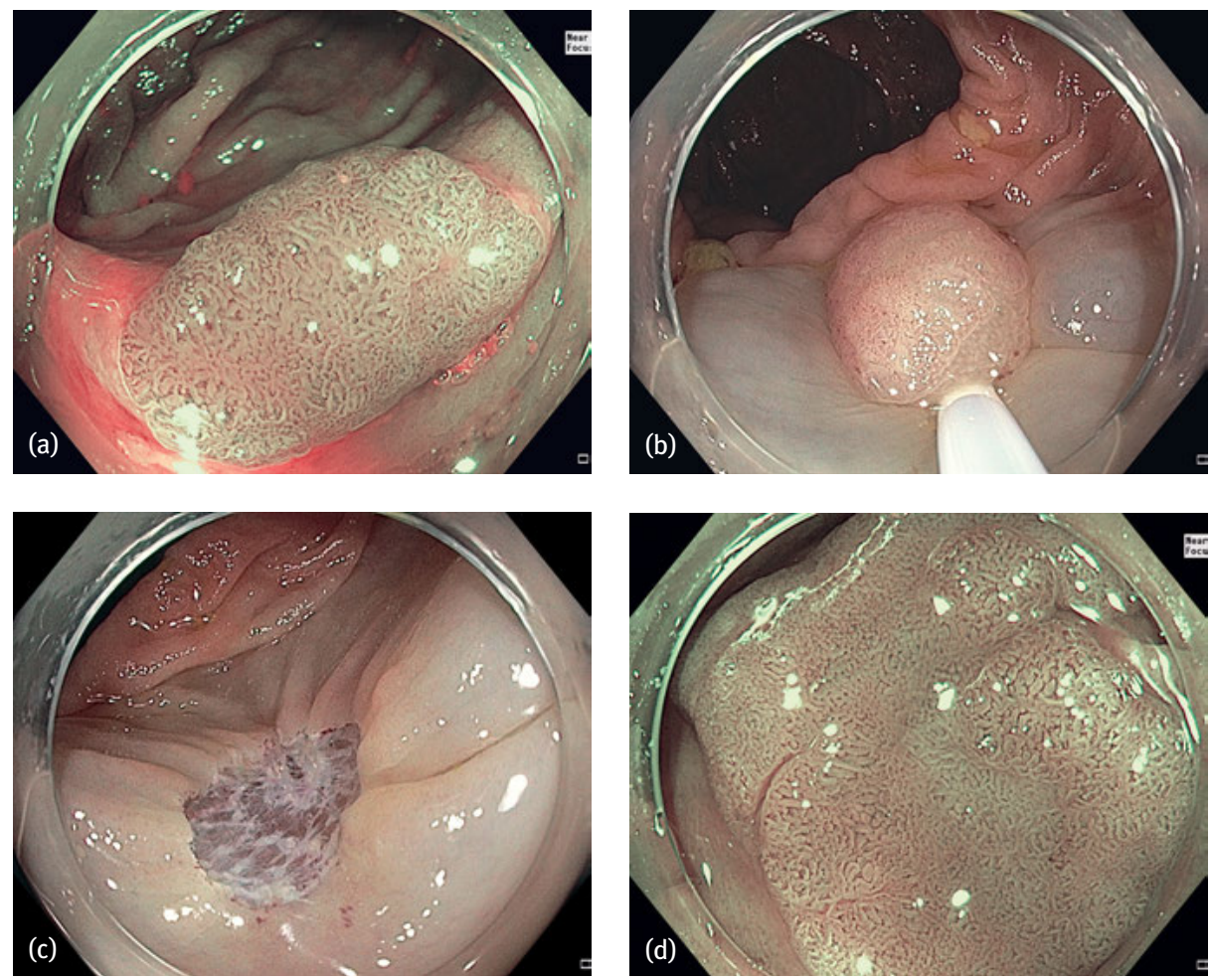

Abb. 31.5: Endoskopische Mukosaresektion (EMR) flacher Kolonpolypen. (a) LST-NG im Kolon transversum (NBI; JNET 2 A, Durchmesser ca. $12 \mathrm{~mm}$ ); (b) platzierte Diathermieschlinge (nach Unterspritzung mit Suprarenin 1:100.000); (c) Resektionsulkus (en bloc-Resektion; Histologie: Adenom mit LGIEN R0); (d) LST-NG im Kolon transversum (NBI; JNET 2 A, Durchmesser ca. 25 mm). 

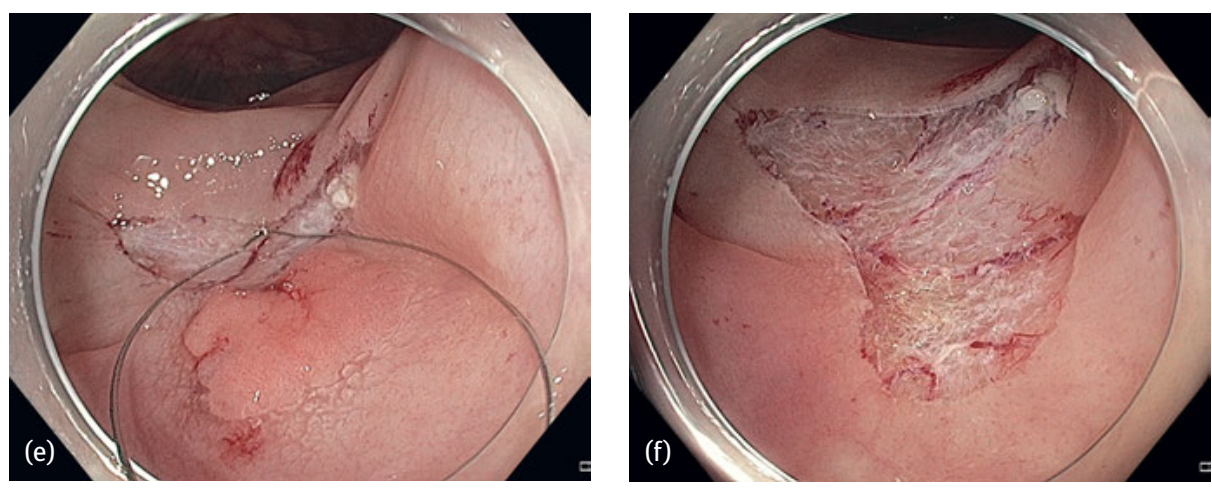

Abb. 31.5: (Fortsetzung) (e) piecemeal-EMR; (f) Resektionsulkus (piecemeal-Resektion; Histologie: Adenom mit LGIEN).

\subsubsection{Endoskopische Submukosadissektion (ESD)}

Als Hauptnachteil nach pEMR gilt das erhöhte Risiko für Rezidive, wobei es sich nahezu immer um wieder gewachsene Reste der initial nicht komplett entfernten Neoplasie handelt. Die Technik der endoskopischen Submukosadissektion (ESD) erlaubt demgegenüber eine Resektion auch großer Läsionen in einem Stück (en blocResektion). Vorteile sind die verbesserte histologische Beurteilung hinsichtlich einer R0-Situation sowie ein nahezu komplett eliminiertes Rezidivrisiko. Im Gegensatz zur EMR erfolgt die Resektion nicht mit Schlingen, sondern mit verschiedenen Messern (Abb. 31.6). Die ESD ist im Vergleich zur EMR technisch anspruchsvoller, zeitaufwändiger, zumindest in unerfahrenen Händen komplikationsträchtig und erfordert eine Lernkurve.

\subsubsection{Endoskopische Vollwandresektion (endoscopic full thickness resection EFTR)}

Sowohl bei der klassischen Polypektomie als auch bei EMR und ESD erfolgt die Resektion innerhalb der Submukosa, während die Muscularis propria intakt bleibt. Zur Resektion der gesamten Kolonwand (Vollwandresektion) ist üblicherweise ein chirurgischer Eingriff erforderlich, der transabdominell oder transanal (für Läsionen des Rektums) erfolgt. Mit dem Full Thickness Resection Device (FTRD; Fa Ovesco, Tübingen, Deutschland) steht seit einigen Jahren ein Instrument zur Verfügung, welches eine endoskopische Vollwandresektion im Kolorektum erlaubt. Die Zielläsion wird inklusive der gesamten Kolonwand in eine auf das Endoskop montierte Kappe hineingezogen, dann durch einen auf der Kappe vorgespannten Metallclip (Over-The-ScopeClip OTSC) gerafft und abschließend das „geclippte“ Gewebe mittels Schlinge rese- 
ziert (Abb. 31.7). Das Verfahren ist auf die Entfernung kleiner Läsionen beschränkt $(<2 \mathrm{~cm})$. Als Hauptindikation gelten vorbehandelte Läsionen, die wegen Vernarbung bzw. non-lifting mit konventionellen Resektionsverfahren nicht mehr endoskopisch therapiert werden können (überwiegend Adenomrezidive nach pEMR).
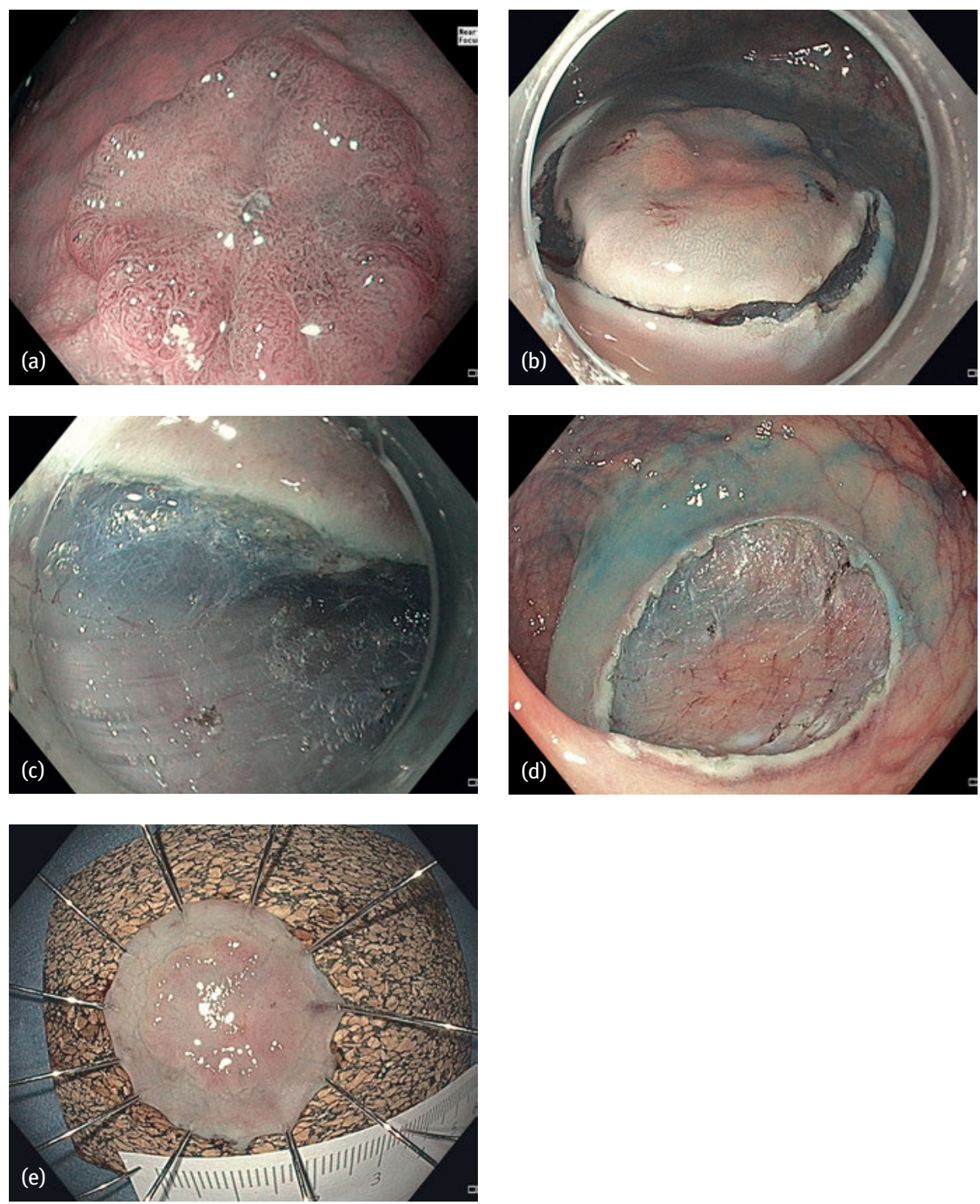

Abb. 31.6: Endoskopische Submukosadissektion (ESD) im proximalen Rektum. (a) LST-NG „pseudo depressed“" (gleiche Läsion wie Abb. 31.2 b) (NBI; JNET 2B, Durchmesser 18 mm); (b) Umschneidung (nach Unterspritzung mit Suprarenin 1:100.000); (c) Dissektion der Submukosa; Blick auf die M. propria, (d) Resektionsulkus; (e) Resektat (auf Korkplatte ausgespannt; Histologie: Adenom mit HGIEN). 

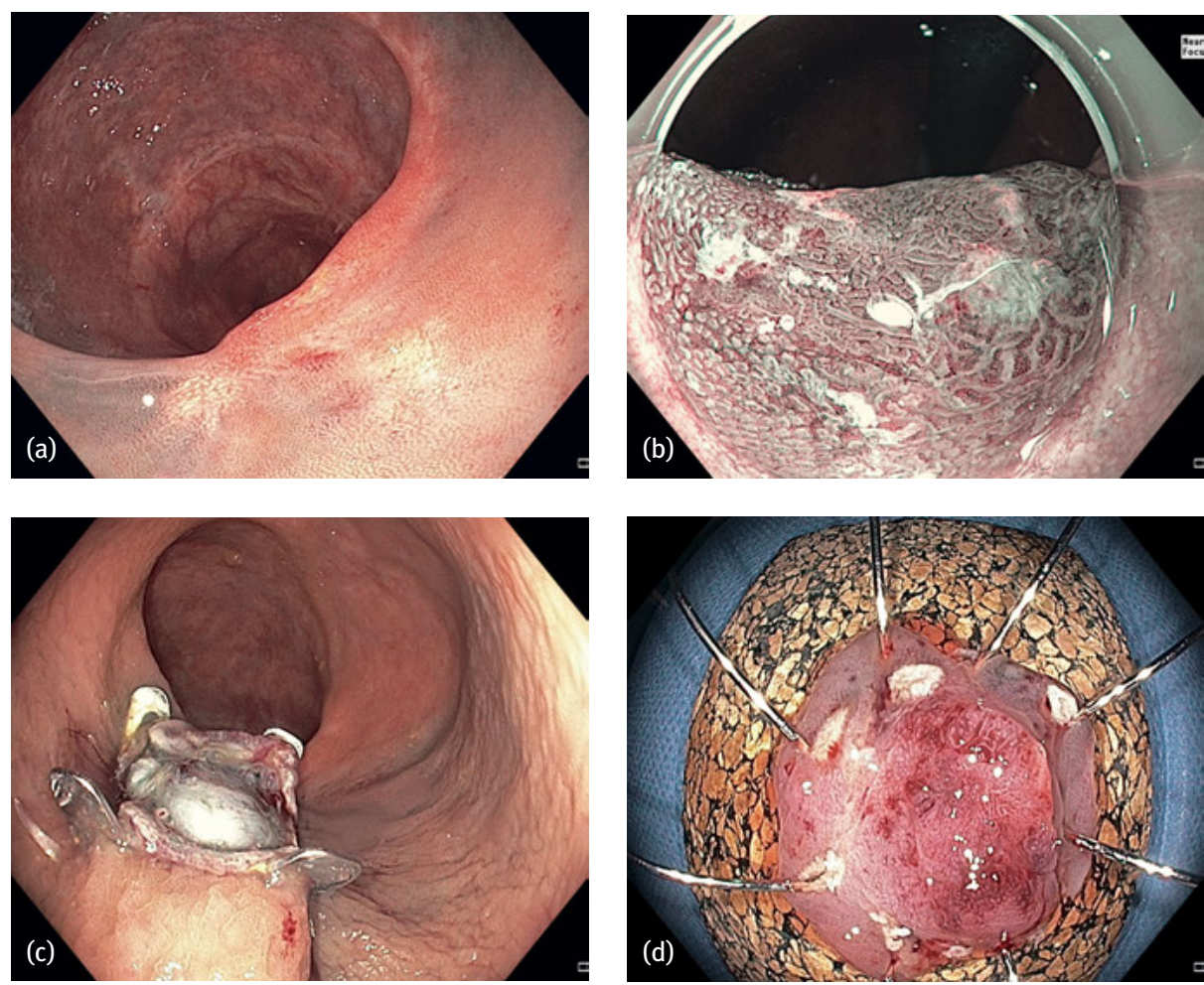

Abb. 31.7: Endoskopische Vollwandresektion (EFTR). (a) Adenomrezidiv auf einer kolorektalen Anastomose $10 \mathrm{~cm}$ ab ano (non-lifting auf der Anastomose sowie durch zusätzliche Tuschemarkierung); (b) NBI: JNET 2B; (c) Resektionsareal nach Vollwandresektion; (d) Resektat (auf Korkplatte ausgespannt; Histologie: Adenom mit HGIEN).

In einer aktuell von Schmidt et al. publizierten Multicenterstudie mit 181 Vollwandresektionen stellten Adenome mit non-lifting (ganz überwiegend Rezidivläsionen) die dominierende Indikation dar; mittels EFTR konnten 77,7 \% dieser Läsionen R0reseziert werden.

\subsection{Endoskopische Resektion - Wahl des Resektionsverfahrens}

Aktuell stellt die EMR das Standardverfahren zur ER kolorektaler Neoplasien mit einem Durchmesser $\geq 10 \mathrm{~mm}$ dar, wenn morphologisch kein Malignomverdacht besteht. Grundsätzlich ist zu klären, bei welchen Läsionen die Vorteile der ESD relevant sind (en bloc Resektion, histologische R0-Diagnose, Rezidivfreiheit) und wann potentielle Nachteile der EMR akzeptiert werden können (R1-Histologie, Rezidive).

Letztlich sollte eine ESD immer dann erwogen werden, wenn eine Ro Resektion unbedingt angestrebt wird, diese aber mit anderen Resektionstechniken (EMR) tech- 
nisch unwahrscheinlich oder nicht möglich erscheint. Dies gilt in erster Linie bei frühmalignen oder malignomsuspekten Läsionen. Die makroskopische Einschätzung des Malignitätspotenzials einer Läsion hat somit entscheidende Bedeutung in der Indikationsstellung zur endoskopischen Resektion und in der Wahl des Resektionsverfahrens (insbesondere EMR versus ESD). Die europäische Endoskopie-Fachgesellschaft (ESGE) hat die Empfehlungen zur endoskopischen Resektion kolorektaler Polypen in einer aktuellen Leitlinie zusammengefasst und empfiehlt bei flachen Läsionen mit einem Durchmesser $\geq 10 \mathrm{~mm}$ vor der Wahl des Resektionsverfahrens ein „advanced endoscopic imaging to identify the presence of submucosal invasion".

\subsubsection{Dignitätsbeurteilung kolorektaler Neoplasien}

Es kann nicht genug betont werden, dass vor der Resektion bzw. vor der Wahl des Resektionsverfahrens alle zur Verfügung stehenden diagnostischen Optionen ausgeschöpft werden sollten, um die Dignität einer Läsion einzuschätzen (Paris-Klassifikation, Chromoendoskopie, digitale Strukturverstärkung).

Die tatsächliche Histologie bzw. Dignität einer Läsion ist oft nicht oder durch vorherige Biopsien nicht zuverlässig bekannt. In einer eigenen Analyse war bei 43 endoskopisch resezierten Rektumfrühkarzinomen das Karzinom nur in 5 Fällen (12\%) durch Biopsie gesichert. Bei weiteren 25 Läsionen (58 \%) war das Karzinom morphologisch vermutet, aber in der Biopsie nicht nachzuweisen und bei weiteren $13(30 \%)$ wurde die Karzinomdiagnose histologisch erst am Resektat bei makroskopisch vorher mutmaßlich als benigne eingestuftem Adenom gestellt.

Die Abschätzung des Malignitätsrisikos muss also anhand morphologischer Kriterien erfolgen, um insbesondere eine pEMR von Frühkarzinomen mit konsekutiver R1-Situation und Rezidivrisiko zu vermeiden.

Als Läsionen mit einem relevanten Malignitätsrisiko gelten LST-G vom „nodular mixed type“ mit großen Knoten (>1 cm) und LST-NG vom „pseudo depressed type“. In einer eigenen Analyse von 300 flächigen Rektumpolypen lag der Anteil submukosainvasiver Karzinome bei 0\% für LST-G vom „homogenous type“, bei 18\% für LST-G vom „,nodular mixed type“ (mit Knoten $>1 \mathrm{~cm}$ ) und bei 73\% für LST-NG vom „pseudo depressed type“. Nach einer ersten Einschätzung hinsichtlich der Wachstumsform sollte eine Feinbeurteilung der Polypenoberfläche erfolgen. Hierzu steht z. B. die NBI-Technologie mit der hierzu entwickelten JNET-Klassifikation zur Verfügung (Tab. 31.1). Als Risikokriterium für eine Submukosainvasion gilt das Stadium 2B der JNET-Klassifikation. Läsionen mit morphologischem Malignitätsrisiko sind in Abb. 31.2, 31.3b, 31.3c, 31.6 und 31.7 dargestellt. Zur Frage einer tiefen Submukosainfiltration und der damit verbundenen Machbarkeit und Sinnhaftigkeit einer ER kann neben der Morphologie die Abhebung einer Läsion nach Unterspritzung herangezogen werden (,lifting sign“). Eine komplett fehlende Abhebung (non-lifting) gilt bei nicht vorbehandelten Läsionen als Hinweis auf eine tiefe Submukosainfiltration. Da eine ER hier zusätzlich 
ein hohes Perforationsrisiko mit sich bringt, empfiehlt die Leitlinie der DGVS bei unvorbehandelten non-lifting-Läsionen die chirurgische Resektion.

Besteht nach Abschluss der morphologischen Diagnostik der Verdacht auf eine maligne Entartung (Submukosainvasion), sollte eine en bloc Resektion mit Abstand nach lateral und zur Tiefe unbedingt angestrebt werden (en bloc, R0). Eine EMR kommt hierbei nur dann in Frage, wenn die Läsion klein ist und mutmaßlich mittels EMR en bloc zu resezieren ist. Ist eine en bloc-Resektion mittels EMR nicht oder nur unsicher möglich, sollte einer ESD oder einer Vollwandresektion der Vorzug gegeben werden. Demgegenüber können die Nachteile der EMR und auch das Rezidivrisiko einer pEMR bei benignen Läsionen eher in Kauf genommen werden. In einer großen australischen Studie mit 1000 Resektionen großer Polypen (pEMR) berichten Moss et al. über eine Rezidivrate von $16 \%$ bei der ersten Kontrolle nach 4 Monaten und zusätzlichen $4 \%$ bei der zweiten Kontrolle nach 16 Monaten. Die Rezidivläsionen waren in mehr als $90 \%$ erneut suffizient endoskopisch therapierbar, so dass im Langzeitverlauf $98 \%$ der Patienten ohne chirurgische Resektion behandelt werden konnten. Die Zahlen unterstreichen neben der Effektivität der EMR auch die unabdingbare Notwendigkeit engmaschiger Kontrolluntersuchungen. Als Risikofaktoren für das Versagen der EMR wurden vorausgegangene Interventionen sowie die Lokalisation an der Ileozökalklappe identifiziert; Hauptrisikofaktor für das Auftreten eines Rezidivs war eine Polypengröße $>4 \mathrm{~cm}$.

Für gestielte Polypen, die mittels Schlingenresektion meist unkompliziert in einem Stück reseziert werden können, existiert die Problematik der prätherapeutischen Dignitätsbeurteilung nicht.

Eine mögliche Vorgehensweise für endoskopische Diagnostik und Wahl des Resektionsverfahrens ist in Abb. 31.8 vorgeschlagen.

\begin{tabular}{|c|c|}
\hline \multicolumn{2}{|c|}{$\begin{array}{l}\text { Beurteilung der Morphologie eines Kolonpolypen } \\
\text { (Paris-Klassifikation? LST-Klassifikation? JNET-Klassifikation?) }\end{array}$} \\
\hline \multicolumn{2}{|r|}{1} \\
\hline \multirow{2}{*}{$\begin{array}{l}\text { Läsion mutmaßlich benigne } \\
(>90 \% \text { aller Läsionen, JNET 1/2A) } \\
\rightarrow \text { Resektionsverfahren in } \\
\text { Abhängigkeit von Polypengröße } \\
<5 \mathrm{~mm} \rightarrow \text { Biopsiezange } \\
6-9 \mathrm{~mm} \rightarrow \text { cold snare } \\
\geq 10 \mathrm{~mm} \rightarrow \text { Domäne der EMR }\end{array}$} & $\begin{array}{l}\text { Risiko für maligne Entartung } \\
\text { ( }<10 \% \text { aller Läsionen, LST-G mit } \\
\text { großem Knoten, LST-NG } \\
\text { „pseudodepressed“, JNET > 2A) } \\
\rightarrow \text { Resektion en bloc anzustreben }\end{array}$ \\
\hline & $\downarrow$ \\
\hline$\nabla$ & $\begin{array}{l}\text { Abschätzung der Invasionstiefe } \\
\text { (oberflächliche vs. tiefe sm-Invasion) }\end{array}$ \\
\hline $\begin{array}{l}\text { v. a. oberflächliche sm-Invasion } \\
\text { (JNET 2B, Abhebung nach } \\
\text { Unterspritzung) } \\
\rightarrow \text { endoskopische en bloc } \\
\text { Resektion (EMR nur, wenn sicher } \\
\text { en bloc möglich, ansonsten ESD) }\end{array}$ & $\begin{array}{l}\text { v. a. tiefe sm-Invasion (JNET 3, keine } \\
\text { Abhebung nach Unterspritzung) } \\
\rightarrow \text { chirurgische Resektion mit } \\
\text { Lymphknotendissektion }\end{array}$ \\
\hline
\end{tabular}

Abb. 31.8: Diagnostik und Therapieentscheidung bei kolorektalen Polypen. 


\subsubsection{Vorgehen beim kolorektalen Frühkarzinom}

Neben der technischen Machbarkeit gilt die onkologische Sinnhaftigkeit als Grundvoraussetzung für eine endoskopische Therapie maligner Kolonpolypen. Diese ist gegeben, wenn eine alleinige ER eine kurative Therapie darstellt und kein Risiko verbliebener Tumorreste oder verbliebener Lymphknotenmetastasen besteht. Die endoskopischen Resektionskriterien für kolorektale Frühkarzinome sind in der DGVSLeitlinie festgelegt. Neben einer histopathologisch dokumentierten R0-Situation muss eine sogenannte „low-risk“-Histologie vorliegen (G1/G2-Läsionen ohne Lymphgefäßeinbruch). Zusätzlich darf eine tiefe Submukosainfiltration nicht vorliegen. Bei gestielten Polypen gilt die histologisch dokumentierte R0-Resektion an der Basis des Stiels als low-risk. Bei flachen Läsionen muss die Submukosainfiltration quantifiziert werden; das Überschreiten einer Submukosainvasionstiefe von mehr als $1000 \mu \mathrm{m}$ gilt heute (noch) als high-risk-Kriterium. Bei Nichterfüllung eines der genannten Kriterien besteht ein Lymphknotenmetastasenrisiko; entsprechend wird eine chirurgische Resektion inklusive Lymphknotendissektion empfohlen. Bei Erfüllung der „low risk“Kriterien wird die ER und die Durchführung endoskopischer Kontrollen nach 6 und 24 Monaten als ausreichend erachtet. Die ESD ist der EMR aufgrund der höheren en bloc-Resektionsrate überlegen und sollte bei der ER frühmaligner Läsionen bevorzugt angewandt werden. In einer vergleichenden Studie von Saito et al. lag die en bloc-Resektionsrate der ESD bei $84 \%$ gegenüber $33 \%$ nach EMR. Die Perforationsrate war nach ESD mit 6,2 vs. 1,3\% erhöht; die Untersuchungsdauer war bei der EMR signifikant kürzer (29 vs. 108 Minuten). Die Rezidivrate war nach ESD mit $2 \%$ signifikant niedriger als nach EMR (14\%). Daten zur kolorektalen ESD sind außerhalb Asiens spärlich. In einer initialen eigenen Studie zur Lernkurve lag die en bloc Resektionsrate bei 81,6\% und die R0 Resektionsrate bei 69,7 \% (ESD bei 82, überwiegend benignen und überwiegend im Rektum gelegenen Läsionen). Mit zunehmender Untersuchungszahl wurden die Ergebnisse besser; nach 50 durchgeführten Resektionen lag die en bloc Resektionsrate bei 96,2\% und die R0 Resektionsrate bei 84,5\%. Die Rezidivrate war erwartungsgemäß nach en bloc Resektion signifikant vermindert (2,9\% vs. $25 \%$ nach piecemeal-Resektion). Beim Rektumfrühkarzinom konnten wir nach Überwindung der Lernkurve die Rate kurativer Resektionen auf 47,6 \% steigern. Hauptursache für nicht-kurative Resektionen war eine sm-Invasion $>1000 \mu \mathrm{m}$. Allerdings waren bei keinem Patienten, die wegen alleiniger Überschreitung der $1000 \mu \mathrm{m}-$ Grenze operiert wurden, Lymphknotenmetastasen nachweisbar. Hinsichtlich der $1000 \mu \mathrm{m}$-Grenze bestehen in den letzten Jahren vermehrt Hinweise, dass möglicherweise auch eine tiefere Submukosainvasion mit einem sehr geringen Lymphknotenmetastasenrisiko assoziiert ist, wenn ansonsten histologische low-risk-Parameter vorliegen (G1/2, L0, V0, kein Tumor-Budding). Hierzu bleiben weitere Daten abzuwarten.

Proximal des Rektums ist die ESD schwierig und weist ein erhöhtes Perforationsrisiko auf. Ursachen sind anatomische Eigenschaften des Kolons (dünne Wand, Falten, Haustren, Flexuren, Peristaltik), die schwierigere Gerätehandhabung und 
die erschwerte Beherrschung möglicher Komplikationen. Eine japanische Multicenterstudie ermittelte das Perforationsrisiko zentrumsabhängig auf 1,9-8,9\% in Institutionen mit mehr als 50 ESDs; demgegenüber standen Perforationsraten bis zu $36 \%$ in Zentren mit weniger als 50 Untersuchungen. In einer kürzlich publizierten deutschen Studie von Sauer et al. wurden neben 63 Rektumpolypen auch 119Läsionen oberhalb des Rektums mittels ESD reseziert; bei akzeptabler Komplikationsrate (9,3\% Mikroperforationen mit endoskopischem Verschluss, 2,7 \% verzögerte Blutung, keine OP-pflichtige Komplikation) wurden R0-Resektionsraten von 70,8 \% bei Läsionen $<50 \mathrm{~mm}$ bzw. von 40,5 \% bei Läsionen $\geq 50 \mathrm{~mm}$ berichtet. Randomisierte Studien zum prospektiven Vergleich von EMR und ESD liegen aktuell nicht vor.

Aktuell kann die ESD proximal des Rektums in Europa derzeit noch nicht als Standardverfahren angesehen werden. Bei frühmalignen oder malignomsuspekten Läsionen sollte die Durchführung einer ESD in einem Zentrum aber bereits heute erwogen werden, wenn eine Abtragung mittels EMR nicht en bloc erfolgen kann.

Die potenzielle Rolle der endoskopischen Vollwandresektion (EFTR) beim Frühkarzinom ist derzeit noch nicht ausreichend einzuschätzen. In der von Schmidt et al. durchgeführten Multicenterstudie mit 181 Vollwandresektionen waren 29Frühkarzinome eingeschlossen, wobei die Rate kurativer Resektionen bei 44,8\% lag. Bei kleinen Frühkarzinomen, nach inkompletter Resektion frühmaligner Läsionen oder aber bei fehlender chirurgischer Option (Komorbidität) kann die EFTR aber durchaus als sinnvolle Option bzw. Reserveverfahren zum Einsatz kommen. Das Risiko einer möglichen Tumorzelldissemination nach intraperitoneal wird kontrovers diskutiert. Bis zum Vorliegen weiterer Daten sollte ein derartiges potentielles Risiko bei der Therapieentscheidung zur EFTR frühmaligner Läsionen bedacht werden.

\subsection{Komplikationen nach ER kolorektaler Polypen}

Hauptkomplikationen der endoskopischen Polypenresektion sind Blutung und Perforation. Jeder Endoskopiker, der Polypenresektionen durchführt, muss das Management dieser Komplikationen beherrschen.

\subsubsection{Blutung}

Die Blutung ist mit einer Häufigkeit von 1-3\% die häufigste Komplikation nach ER kolorektaler Polypen. Sie kann während oder unmittelbar nach der ER, aber auch verzögert innerhalb der ersten Tage und Wochen nach der Intervention auftreten. Hauptrisikofaktoren für eine Blutung sind die Größe und Zahl der entfernten Polypen sowie eine bestehende Medikation mit Thrombozytenaggregationshemmern bzw. Antikoagulantien. In einer großen Studie beschrieben Albeniz et al. eine Blutungsrate von 3,7 \% nach Abtragung flacher Polypen mit einem Durchmesser $>20 \mathrm{~mm}$. Ri- 
sikofaktoren waren ein Patientenalter $\geq 75$ Jahre, ASA-Score III-IV, Aspirineinnahme, Lokalisation im rechten Hemikolon, Polypendurchmesser $\geq 40 \mathrm{~mm}$ sowie fehlender Clipverschluss der Abtragungsfläche. Bereits während bzw. am Ende der ER können präventive Maßnahmen zur Blutungsprophylaxe erwogen werden (Clipapplikation, Koagulation von Gefäßen mit der Spitze der verwendeten Diathermieschlinge oder einer Koagulationszange). Kommt es trotzdem zur Blutung, stehen prinzipiell alle Verfahren zur Verfügung, die auch bei anderen gastrointestinalen Blutungen zum Einsatz kommen. Am häufigsten werden die Injektionstherapie und die Clipapplikation eingesetzt.

\subsubsection{Perforation}

Die Perforation ist die schwerwiegendste Komplikation während bzw. nach ER im Kolon. In einer großen japanischen Studie betrug das Gesamtrisiko 0,74 \% (0,17 \% nach Polypektomie, 0,91 \% nach EMR und 3,3\% nach ESD). Als Risikofaktoren gelten Polypen-assoziierte Faktoren (Größe, Lage, Rezidivsituation, lifting), die Art des Resektionsverfahrens und im Wesentlichen die Expertise des Endoskopikers. Relevant ist der Zeitpunkt der Perforation. Bei einer bereits während der Intervention auftretenden und auch bemerkten Perforation sollte ein endoskopischer Verschluss immer angestrebt werden. Meist ist dies durch Applikation von Metallclips möglich (Abb. 31.9). Gelingt ein endoskopischer Verschluss, kann unter konservativer Therapie mit intravenöser Flüssigkeitszufuhr, Antibiotikagabe und initialer Nahrungskarenz zunächst zugewartet werden. Engmaschige klinische Kontrollen unter stationären Bedingungen sind unabdingbar.

Eine verzögerte Perforation wird nach einem beschwerdefreien Intervall durch klinische Symptome erkennbar (Schmerzen, Fieber, Peritonismus). Ursächlich ist oft eine verzögerte Nekrose einer durch Koagulation geschädigten M. propria im Resek-
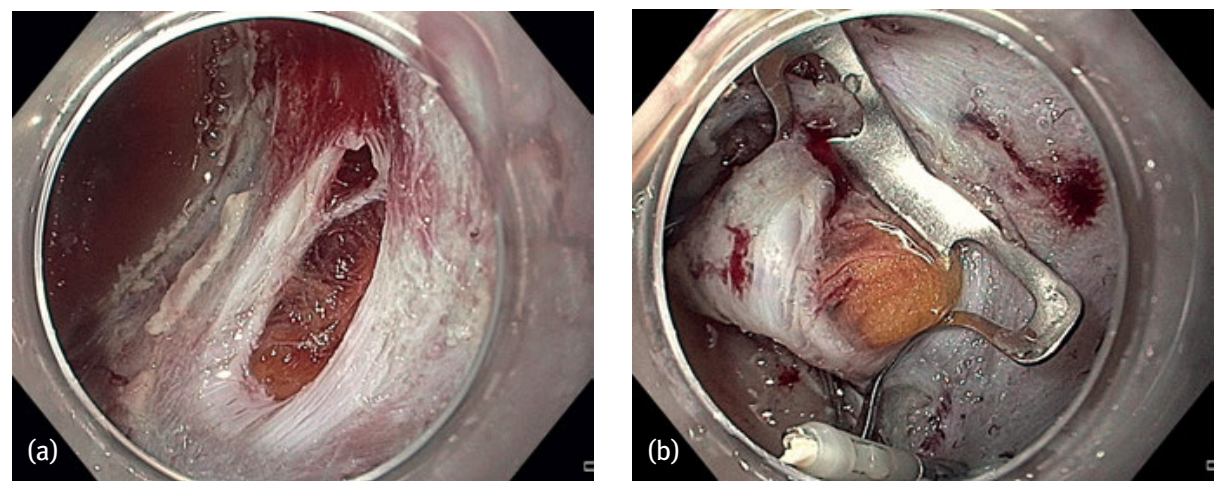

Abb. 31.9: Komplikationsmanagement nach ER im Kolon. (a) Perforation im Rahmen eine pEMR;

(b) Clipverschluss mittels OTS-Clip. 
tionsareal. Eine umgehende radiologische Diagnostik sollte zur Diagnosesicherung erfolgen (möglichst mit CT). Gleiches gilt, wenn sich nach mutmaßlich suffizientem initialem Clipverschluss im Verlauf eine klinische Verschlechterung entwickelt. Bestätigt sich in der Computertomographie die Perforation, muss bei der verzögerten Perforation in aller Regel chirurgisch vorgegangen werden. Findet sich freie intraabdominelle Luft ohne klinische Symptomatik (z. B. als Zufallsbefund bei radiologischer Diagnostik aus anderer Indikation), kann meist abgewartet werden. Prinzipiell muss das Vorgehen nach jeder Perforation interdisziplinär zwischen Endoskopiker und Viszeralchirurgen abgesprochen werden; eine umgehende Information des Viszeralchirurgen während bzw. nach der Endoskopie ist anzustreben.

\subsection{Nachsorge nach ER kolorektaler Polypen}

Nach piecemeal-Resektion benigner neoplastischer Kolonpolypen besteht ein erhöhtes Risiko für Lokalrezidive; die deutsche Leitlinie empfiehlt deshalb eine Kontrollkoloskopie nach 2-6 Monaten. Bei sicher polypenfreiem Kolon werden Kontrollkoloskopien zur Früherkennung metachroner Neoplasien empfohlen. Das Intervall hängt hierbei von der Ausgangssituation ab. Bei unauffälliger Koloskopie wird die Folgeuntersuchung nach 10 Jahren empfohlen. Nach Polypenresektion entscheidet die Anzahl und die Risikomorphologie der entfernten Polypen über das Kontrollintervall (5-10 Jahre bei 1-2 low-risk-Polypen, 3 Jahre bei 3-4 Polypen oder einem high-riskPolypen, < 3 Jahre bei $\geq 5$ Polypen). Als high-risk-Kriterien gelten bei benignen Polypen ein Durchmesser $\geq 1 \mathrm{~cm}$, eine villöse Histologie und eine schwere intraepitheliale Neoplasie (HGIEN). Nach kurativer endoskopischer Entfernung kolorektaler Frühkarzinome wird eine endoskopische Kontrolle der Abtragungsstelle nach 6 Monaten und eine komplette Koloskopie nach 3 Jahren empfohlen.

\section{Weiterführende Literatur}

Albeniz E, Fraile M, Ibanez B, et al. A Scoring System to Determine Risk of Delayed Bleeding After Endoscopic Mucosal Resection of Large Colorectal Lesions. Clin Gastroenterol Hepatol. 2016;14:1140-1147.

Ferlitsch M, Moss A, Hassan C, et al. Colorectal polypectomy and endoscopic mucosal resection (EMR): European Society of Gastrointestinal Endoscopy (ESGE) Clinical Guideline. Endoscopy. 2017;49:270-297.

Kudo S. Endoscopic mucosal resection of flat and depressed types of early colorectal cancer. Endosocpy. 1993;25:455-461.

Kudo S, Tamura S, Nakajima T, et al. Diagnosis of colorectal tumorous lesions by magnifying endoscopy. Gastrointest Endosc. 1996;44:8-14.

Lambert R for the Endoscopic Classification Review Group. Update on the Paris Classification of Superficial Neoplastic Lesions in the Digestive Tract. Endoscopy. 2005;37:570-578. 
Leitlinienprogramm Onkologie (Deutsche Krebsgesellschaft, Deutsche Krebshilfe, AWMF): S3-Leitlinie Kolorektales Karzinom, Langversion 2.0, 2017, AWMF Registrierungsnummer: 021-0070L. http://leitlinienprogramm-onkologie.de/Leitlinien/kolorektales-karzinom

Moss A, Williams SJ, Hourigan LF, et al. Long-term adenoma recurrence following wide-field endoscopic mucosal resection (WF-EMR) for advanced colonic mucosal neoplasia is infrequent: results and risk factors in 1000 cases from the Australian Colonic EMR (ACE) study. Gut. 2015;64:57-65.

Moss A, Bourke MJ, Williams SJ, et al. Endoscopic Mucosal Resection Outcomes and Prediction of Submucosal Cancer From Advanced Colonic Mucosal Neoplasia. Gastroenterology. 2011;140:1909-1918.

Probst A, Ebigbo A, Märkl B, et al. Endoscopic submucosal dissection for early rectal neoplasia: experience from a European center. Endoscopy. 2017;49:222-232.

Probst A, Golger D, Anthuber M, et al. Endoscopic submucosal dissection in large sessile lesions of the rectosigmoid: learning curve in a European Center. Endoscopy. 2012;44:660-667.

Saito Y, Fukuzawa M, Matsuda T, et al. Clinical outcome of endoscopic submucosal dissection versus endoscopic mucosal resection of large colorectal tumors as determined by curative resection. Surg Endosc. 2010;24:343-352.

Sano Y, Tanaka S, Kudo SE, et al. NBI magnifying endoscopic classification of colorectal tumors proposed by the Japan NBI Expert Team (JNET). Dig Endosc. 2016;28:526-533.

Sauer M, Hildenbrand R, Oyama T, et al. Endoscopic submucosal dissection for flat or sessile colorectal neoplasia $>20 \mathrm{~mm}$ : A European single-center series of 182 cases. Endosc Int Open. 2016;4:E895-E900.

Schmidt A, Beyna T, Schumacher B, et al. Colonoscopic full-thickness resection using an over-thescope device: a prospective multicentre study in various indications. Gut. 2018;67:1280-1289.

Uraoka T, Saito Y, Matsuda T, et al. Endoscopic indications for endoscopic mucosal resection of laterally spreading tumors in the colorectum. Gut. 2006;55:1592-1597. 
